Current Pathway

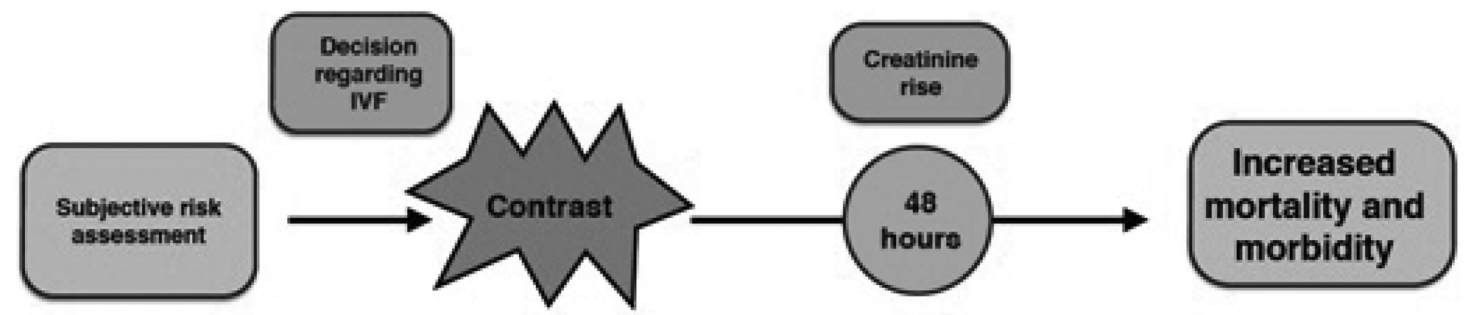

Proposed Pathway

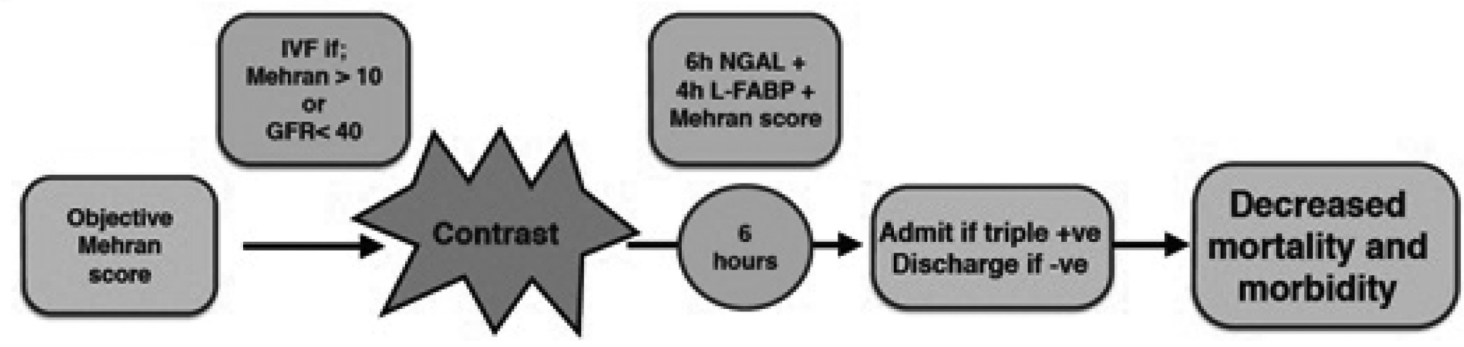

Abstract 208 Figure 2 Proposed patient pathways

Histiocytoid cardiomyopathy (CM) is a rare, distinctive form of cardiomyopathy, characterised by malignant arrhythmias and associated sudden death. $\sim 90 \%$ of cases present in females $<2$ years of age. We undertook whole exome sequencing (WES) in five unrelated affected females, including one with parental samples available. In the family trio we identified a de novo nonsense mutation in NDUFB11 (c.262C>T; p. Arg88*), located on the $\mathrm{X}$ chromosome and encoding a component of the mitochondrial respiratory chain (MRC). Mutations in NDUFB11, including one identical to the one we describe here, have been reported to cause microphthalmia and linear skin defects syndrome (MLS). During the course of our studies, additional mutations in NDUFB11 were associated with Histiocytoid CM by another group. Four of the affected individuals in our study did not carry variants in NDUFB11. Heterozygous mutations in HCCS (which encodes an important mitochondrially-targeted protein) and $C O X 7 B$ (which, like NDUFB11, encodes a protein of the MRC) have also previously been identified in MLS syndrome including a case with features of both MLS syndrome and Histiocytoid CM. However, a systematic review of WES data from previously published cases, alongside the four additional cases presented here, did not identify any further mutations in these genes in Histiocytoid CM. We conclude thattruncating variants in NDUFB11 link the distinct phenotypes of Histiocytoid CM and MLS syndrome. Screening for malignant arrhythmias and cardiomyopathy would be appropriate in individuals with MLS syndrome. Additional nuclear encoded mitochondrial or mitochondrial DNA genes are good candidates for further causes of both Histiocytoid CM and MLS syndrome.

\section{CLINICAL UTILITY OF GENE PANEL AND CLINICAL EXOME TESTING IN CARDIAC DISEASE}

${ }^{1}$ Mary Gable*, 'Chris Buxton, 'Geoff Woodward, 'Marc Wadsley, 'Julie Honeychurch, ${ }^{1}$ Davies Joanne, ${ }^{2}$ Ruth Newbury-Ecob, ${ }^{2}$ Karen Low, ${ }^{2}$ Alan Donaldson, ${ }^{2}$ Josphine Affleck, ${ }^{1}$ Rebecca Whittington, ${ }^{1}$ Mark Greenslade, 'Maggie Williams. 'Bristol Genetics Laboratory; ${ }^{2}$ Department of Clinical Genetics; *Presenting Author

\subsection{6/heartjnl-2016-309890.210}

Cardiac disease is genetically heterogeneous with genes associated with multiple cardiac diseases, multiple causal genes per disease, and often multiple variants in one or more genes contributing to disease presentation. Gene panel testing, either through a specific targeted design, or by virtual analysis from the exome/clinical exome is an ideal approach for genetic diagnosis and provides information regarding complexity of these diseases.

Bristol Genetics Laboratory provides a targeted gene panel for paediatric cardiomyopathy (PC) (71 genes, Agilent SureSelect) and a variety of virtual gene panels from Agilent Focussed Exome; Congenital Heart Defect (CHD) (38 genes), Aortopathy (28 genes), Arrhythmia (54 genes), Cardiomyopathy (119 genes), Connective Tissue (42 genes) Molecular Autopsy (208 genes) and bicuspid aortic valve (8 genes). Whole clinical exome analysis (6110 genes) with phenotypic prioritisation of variants based on HPO terminology using Exomiser can also be performed for patients with complex phenotypes whose phenotype does not clearly fit into a predefined gene panel or for patients who tested negative for a specific panel. 
133 patients have been tested, including newly diagnosed cardiac cases, patients negative for other cardiac gene testing, and patients who have phenotype/genotype incompatibility where contribution of more than one gene is suspected. 41/ $133(30 \%)$ patients have at least one potentially pathogenic variant. 22 patients have multiple plausible variants.

We present data from the cardiac cohort tested to date and cases illustrating the utility and complexity of gene panel testing for cardiac disease including; 1) A paediatric patient with Left Ventricular Non-Compaction (LVNC), dilated aortic root and sinus brachycardia heterozygous (on the PC 71 gene panel) for a novel TMEM43 variant c.994A $>$ G, p. (Thr332Ala) of unknown clinical significance. Further testing using a bespoke 138 gene cardiac panel from the Focused Exome detected a novel splice variant in the HCN4 gene associated with LVNC and primary sinus brachycardia (Milano et al, 2014). This patient's mother who has aortic dilation and regurgitation was heterozygous for the TMEM43 variant and the half-brother who also has dilated aortic root and LVNC did not carry either variant. 2) A large Dilated Cardiomyopathy (DCM) family with variable severity between family members, one affected cousin was heterozygous for a variant of uncertain significance in MYBPC3 c.3384G>C, p. (Glu1128Asp) and another affected cousin heterozygous for a truncating TTN variant, c.89244del, p.(Phe29748Leufs*7). Further family studies are ongoing.

Detailed phenotypic assessment (using a clinical proforma) has been shown to increase diagnostic yield in patients with complex cardiac disease.

\section{REFERENCE}

1 Milano et al. J Am Coll Cardiol 2014;64(8):745-56

\section{NON-CANONICAL NF-ÎŠB SIGNALLING PROMOTES ENDOTHELIAL PROLIFERATION IN RESPONSE TO LOW WALL SHEAR STRESS}

Neil Bowden*, Shuang Feng, Sheila Francis, Paul Evans. University of Sheffield; *Presenting Author

\subsection{6/heartjnl-2016-309890.211}

Introduction Atherosclerotic plaques are predominantly localised to bends and branches of arteries. In contrast to straight regions where the blood exerts unidirectional high frictional drag, known as wall shear stress (WSS) on endothelial cells (ECs), at atheroprone sites ECs are exposed to complex multidirectional low WSS. These forces cause altered gene expression in ECs, leading to enhanced proliferation, apoptosis, inflammation and lesion development. While the canonical NF-I $\mathrm{O} B$ pathway has been well studied in early atherogenesis, the non-canonical NF-Î̃o $\mathrm{B}$ pathwayâ€" regulated by proteaso-

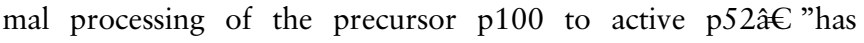
not been examined directly in this context.

Methods and results To determine the effect of WSS on noncanonical NF-Î $\mathrm{B}$ activity, human umbilical vein ECs (HUVECs) were exposed to $72 \mathrm{~h}$ of low or high WSS and non-canonical NF-I- $\mathrm{O}$ expression was analysed by Western blotting. Levels of p100, p52, RelB and IKKÎ \pm were elevated under low WSS in comparison to high WSS. In addition, exposure to a known non-canonical NF-Î$-\mathrm{B}$ stimulus CD40L revealed exaggerated pathway activity under low WSS. Depletion of p100 by siRNA resulted in a decrease in proliferation in response to low WSS, measured by Western blotting for Ki67 and staining for PCNA.

Conclusions The non-canonical NF- $\hat{\mathrm{I}} \mathrm{o} \mathrm{B}$ pathway is primed by low WSS for enhanced activation in response to physiological stimuli. p100/p52 promoted EC proliferation under low WSS conditions, indicating that this pathway could be targeted in the prevention or treatment of atherosclerosis.

\section{ASSESSMENT OF LEFT VENTRICULAR CONTRACTILE RESERVE IN PATIENTS WITH SEVERE SYMPTOMATIC AORTIC STENOSIS AND PRESERVED EJECTION FRACTION}

Ana Rita Cabaco*, Omar Aldalati, Mehdi Eskandari, Miriam Silaschi, Emma Alcock, Jonathan Byrne, Olaf Wendler, Mark Monaghan, Ajay Shah, Philip MacCarthy, Rafal Dworakowski. King's College London BHF Centre of Excellence and King's College Hospital; *Presenting Author

\subsection{6/heartjnl-2016-309890.212}

Introduction Transcatheter aortic valve implantation (TAVI) has become the standard of care for high risk patients. Perioperative deterioration of left ventricular (LV) contractile function was previously demonstrated after surgical aortic valve replacement. Moreover, there is evidence to suggest that patients with severe LV hypertrophy have diminished contractile reserve. We sought to compare the contractile reserve of aortic stenosis (AS) patients to control and heart failure groups utilising a gold standard load-independent technique.

Methods Patients undergoing TAVI under general anesthesia (AS) and control (Ctrl) and heart failure (HF) patients undergoing diagnostic coronary angiography were recruited for invasive pressure-volume loop studies. We measured systolic indices at rest and followed that by assessment of force-frequency relations with atrial pacing. After correction to body surface area, linear mixed model analysis and Friedman test were used to identify differences. Mean values and standard errors are reported.

Results Sixteen (16) patients were in AS group Vs 15 and 12 in Ctrl and HF groups (age in years $84.3 \pm 1.5$, Vs $59.4 \pm$ 2.2 and $48.5 \pm 4.2, \mathrm{p}<0.001$; male $8(50 \%)$, Vs $5(33 \%)$ and $8(66 \%), p=0.46$, respectively).

At rest, ejection fraction (EF) for AS group was $66 \% \pm 4$ Vs $64 \% \pm 4,42 \% \pm 5$ for Ctrl and HF respectively ( $\mathrm{p}=$ 0.006). The maximum first derivative of $\mathrm{LV}$ pressure $(\mathrm{dP} /$ $\left.\mathrm{dt}_{\max }\right)$ was $1097 \pm 77 \mathrm{mmHg} / \mathrm{s}$ Vs $1327 \pm 63$ and $1034 \pm$ $58, \mathrm{p}=0.017$. The load-independent parameters included end-systolic pressure volume relationship (ESPVR) for AS 1.85 $\pm 0.25 \mathrm{mmHg} / \mathrm{ml} \mathrm{Vs} 1.95 \pm 0.35,1.06 \pm 0.18(\mathrm{p}=0.14)$, Starling Contractile Index (SCI) for AS $7.06 \pm 0.9 \mathrm{mmHg} / \mathrm{ml} /$ $\mathrm{s}$ Vs $7.34 \pm 0.9,4.77 \pm 0.6(\mathrm{p}=0.124)$ and Preload Recruitable Stroke Work (PRSW) for AS $40 \pm 4.2 \mathrm{mmHg} / \mathrm{ml}$ Vs $39.6 \pm 4.9,22 \pm 3.7$ for Ctrl and HF respectively $(\mathrm{p}=$ $0.03)$.

With incremental pacing, $\mathrm{dP} / \mathrm{dt}_{\max }$ was biphasic in $\mathrm{AS}$ patients (1097 to 1300 then $1084, \mathrm{p}=0.24$ ) but upsloping in Ctrl cohort (1327 to $1778, \mathrm{p}=0.045)$ and flat in $\mathrm{HF}$ (1034 to $1356, \mathrm{p}=0.19$ ) (Figure 1 ). ESPVR declined steadily in AS patients with incremental pacing unlike the other 2 groups however the changes did not reach statistical significance. SCI response was biphasic in AS (7.7 to 11.9 then 8.1, $\mathrm{p}=0.18)$, upsloping in both Ctrl group (5.6 to $11, \mathrm{p}<$ 0.01 ) and HF cohort (4.5 to 6.1, $\mathrm{p}=0.006$ ) (Figure 2). 